\title{
Integrated Computational Materials Engineering: A Multi-Scale Approach
}

\author{
NITIN CHOPRA ${ }^{1,2,3}$ \\ 1.-Department of Metallurgical and Materials Engineering, The University of Alabama, \\ Tuscaloosa, AL 35487, USA. 2.-Department of Biological Sciences, The University of Alabama, \\ Box 870202, Tuscaloosa, AL 35487, USA. 3.—e-mail: nchopra@eng.ua.edu
}

Computational materials science and engineering has a strong potential for developing robust simulation-based designs for optimizing materials, components, and engineering structures. Integrated computational materials engineering (ICME) provides practical solutions for engineering design tasks and has been recognized by the National Academy of Engineering (NAE Report 2008). ${ }^{1}$ The vital role of materials in design as well as the importance of computation in materials engineering necessitate understanding structure, properties, processing, and performance of materials and designed components. Thus, multiscale modeling has extended into solid mechanics, fluid mechanics, materials science, physics, mathematics, biology, and chemistry. ICME couples quantum scales with macroscales. It also allows the integration of materials processing and product performance for simulation-based manufacturing. ${ }^{1}$ These advances are analogous to transistor models that have led to complex logic operations and concurrent multilevel simulations. ${ }^{1}$ ICME can reduce product development time and costs by minimizing design cycles and facilitating cost-effective design optimization. These benefits have the potential to drive the utilization of multiscale modeling in various industrial sectors.

The "integrated" part of ICME strongly emphasizes synergistic systems and interdisciplinary thinking accompanied by multiscale modeling methods. ${ }^{2,3}$ However, a key challenge is to link the various disciplines. ${ }^{1,4}$ Mathematics and statistics play a critical role and form a strong basis for modeling strategies. The terms "bottom-up" and "top-down" are of particular interest. ${ }^{1}$ The following characteristics are critical for these approaches: (I)

Nitin Chopra is the Guest Editor for the ICME Committee of the TMS Materials Processing \& Manufacturing Division, and coordinator of the topic Multi-Scale Modeling: Concurrent and Hierarchical Methods in this issue. length scales, (II) degrees of freedom and boundary conditions, (III) geometric and energy constraints, (IV) suitable numerical model implementation, (V) determining right sequence of calculations to be performed, (VI) determining variables and equations describing structure-property function, (VII) analyzing the cause-effect relationships using numerical methods, (VIII) verifying effects by experimental approaches before using the developed theory for next higher scales, and (IX) estimating the uncertainty in simulations and experiments to help develop a concrete multiscale modeling approach.

This issue of JOM presents nine invited research and review articles. These articles discuss the importance of the ICME approach in refining conventional material and component processing methods as well as advanced materials processing. The article by Ghosh et al. discusses multiscale modeling of heterogeneous metals and alloys. They comprehensively address the problems related to hierarchical-concurrent multilevel or multiscale modeling methods for ductile fracture and nested dual-stage homogenization method for microstructure-based models for cast $\mathrm{Al}$ alloys. The two articles by Horstemeyer and colleagues detail the development and deployment of the modified embedded atom method (MEAM). The authors focus on the MATLAB-based MEAM potential calibration tool and interface with LAMMPS. The technique is used for studying aluminum system. To further define the ICME in "completeness," the article by Liu et al. discusses case studies focused on three examples, where experiments are integrated with computation aspects. The modeling methods described include artificial neural networks, dislocation dynamics, and CALPHAD approach. Asadi et al. discuss the phase-field crystal model and multiscale analysis of solid-liquid structures. The article also compares various phase-field models for calculating solid-liquid interfaces. Bryan et al. demonstrate alloy design using the ICME-based 
modeling approach, where thermodynamic and kinetic parameters are related to underlying materials physics. The article by Hassan et al. investigates the structural and thermomechanical behavior of steel potshell under different cooling techniques. The results are supported by mechanical testing, microscopic analysis, and thermal behavior analyses. The thermal and mechanical aspects of potshell enable a three-dimensional (3-D) thermomechanical finite-element model for the pot. Overall, the study highlights mechanical integrity of pots required for smelter applications. Guvenc et al. apply the ICME approach to an automobile crash box composed of manganese steel. The mechanical integrity of the crash box was studied in a drop tower test, and the results were validated by simulations (ab initio methods). This also helped in estimating the energyabsorption capacity. In contrast to ICME approaches suitable to conventional material systems and processing methods, the article by Bunn et al. demonstrates the ICME approach to study the composition and thickness profiles of sputtered thin films.

Thus, this special topic of $J O M$ presents a variety of ICME routes involving multiscale phenomena and analysis to design robust material systems. These articles collectively answer the questions pertaining to the advancements in the field of ICME with researchers working not only in conventional material design but also in exotic materials and properties. The future of ICME is very promising. In this regard, significant breakthroughs in the knowledge base, software integration, length and time scale bridging, and educational enterprise are required.

The following papers being published under the topic of Multi-Scale Modeling: Concurrent and Hierarchical Methods provide excellent details and research on the subject. To download any of the papers, go to http://link.springer.com/journal/11837/ $67 / 1 /$ page/ 1 to the Table of Contents page for the January 2015 issue (vol. 67, no. 1).

- "Adaptive Hierarchical-Concurrent Multi-Scale Modeling of Ductile Failure in Heterogeneous Metallic Materials," by Somnath Ghosh

- "Hierarchical Bridging Between Ab Initio and Atomistic-Level Computations: Calibrating the
Modified Embedded Atom Method (MEAM) Potential: Part A," by M.F. Horstemeyer, J.M. Hughes, N. Kukhija, W.B. Lawrimore II, S. Kim, R. Carino, and M.I. Baskes

- "Hierarchical Bridging Between Ab Initio and Atomistic-Level Computations: Sensitivity and Uncertainty Analysis for the Modified Embedded Atom Method (MEAM) Potential: Part B," by J.M. Hughes, M.F. Horstemeyer, R. Carino, N. Sukhija, W.B. Lawrimore II, S. Kim, and M.I. Baskes

- "Discovery via Integration of Experimentation and Modeling: Three Examples for Titanium Alloys," by Y. Liu, P. Samimi, I. Ghamarian, D.A. Brice, D.E. Huber, Z. Wang, V. Dixit, S. Koduri, H.L. Fraser, and P.C. Collins

- "A Review on Quantitative Phase-Field Crystal Modeling of Solid-Liquid Structures," by Ebrahim Asadi and Mohsen Asle Zaeem

- "Concurrent Integration of Science-Based Mechanistic Relationships with Computational Thermodynamics and Kinetic Simulations for Strengthening Magnesium Alloys at Elevated Temperatures," by Z.L. Bryan and M.V. Manuel

- "Multiple-Scale Thermomechanical Analysis of a Forced-Cooled Aluminum Reduction Pot," by Mohgamed I. Hassan, Ayoola T. Brimmo, and Youssef Shatilla

- "ICME for Crashworthiness of TWIP Steels: From $\mathrm{Ab}$ Initio to the Crash Performance," by O. Güvenç, F. Roters, T. Hickel, and M. Bambach

- "A Semiempirical Model for Tilted-Gun Planar Magnetron Sputtering Accounting for Chimney Shadowing," by J.K. Bunn, C.J. Metting, and J. Hattrick-Simpers

\section{REFERENCES}

1. M.F. Horstemeyer, Integrated Computational Materials Engineering (ICME) for Metals: Reinvigorating Engineering Design with Science (New York: Wiley, 2012).

2. S. Yip, Handbook of Materials Modeling (Dordrecht: Springer, 2005).

3. M.F. Horstemeyer, Practical Aspects of Computational Chemistry, ed. J. Leszczynski and M.K. Shukla (New York: Springer, 2009), pp. 87-135.

4. E.B. Tadmor, R. Phillips, and M. Ortiz, Int. J. Solids Struct. 37,379 (2000). 